\title{
Efficacy and safety of tyrosine kinase inhibitors in advanced non-small-cell lung cancer harboring epidermal growth factor receptor mutation: a network meta-analysis
}

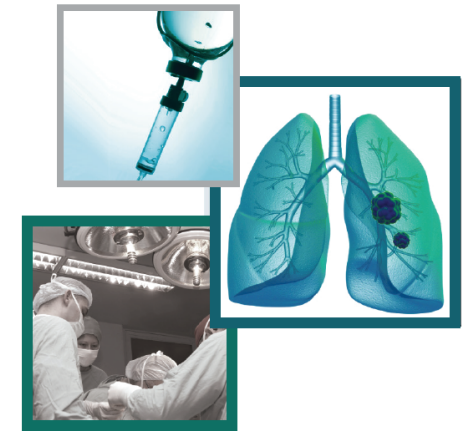

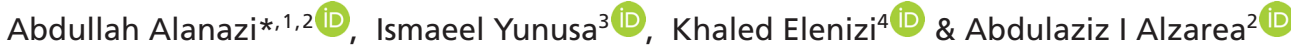 \\ ${ }^{1}$ Department of Pharmaceutical Economics \& Policy, School of Pharmacy, Massachusetts College of Pharmacy and Health Sciences \\ (MCPHS), Boston, MA 02115, USA \\ ${ }^{2}$ College of Pharmacy, Jouf University, Sakaka, Aljouf 72341, Saudi Arabia \\ ${ }^{3}$ University of South Carolina College of Pharmacy, Columbia, SC 29208, USA \\ ${ }^{4}$ Department of Internal Medicine College of Medicine, Prince Sattam bin Abdulaziz University, Alkharj 11942, Saudi Arabia \\ *Author for correspondence: Asdalananzi@ju.edu.sa
}

\begin{abstract}
Aim: To compare the efficacy and safety of tyrosine kinase inhibitors (TKIs) as first-line treatment in patients with locally advanced or metastatic non-small-cell lung cancer (NSCLC) with positive EGFR mutation. Materials \& methods: Following a systematic literature review until December 2019, we conducted a random-effects pairwise and network meta-analyses (NMA). We ranked treatments for efficacy and safety based on the surface under the cumulative ranking curve (SUCRA). Results: Tyrosine kinase inhibitors of the epidermal growth factor receptor (EGFR-TKI) improved survival outcomes with fewer grade 3 or higher adverse events compared to chemotherapy. Overall survival results suggest that osimertinib has the highest probability of being the most efficacious (SUCRA, 79.9\%), followed by dacomitinib (SUCRA, 75.8\%). Adverse events results suggest that osimertinib (SUCRA, 84.3\%) and gefitinib (SUCRA, 78.9\%) has the highest probability of being the safest. Conclusion: In this NMA, we found that osimertinib is the most efficacious and safest EGFR-TKI. These results may guide clinicians in choosing the most appropriate treatment option among EGFR-TKIs for their patient's individual clinical characteristics.
\end{abstract}

First draft submitted: 27 March 2020; Accepted for publication: 26 October 2020; Published online: 23 November 2020

Keywords: epidermal growth factor receptor • lung cancer • network meta-analysis • tyrosine kinase inhibitors

\section{Background}

Lung cancer has the highest mortality rate among all cancer types, with a 5-year survival rate of $18.6 \%$ in the US [1]. Non-small-cell lung cancer (NSCLC) represents roughly $85 \%$ of all lung cancer cases [2]. The majority of patients diagnosed with advanced NSCLC are unsuitable for surgery or radiotherapy [3]. First-line platinum-based doublet chemotherapy used to be the standard of care for patients with advanced NSCLC, according to the American Society of Clinical Oncology and European Society for Medical Oncology clinical practice guidelines [4,5]. In the last decade, several genetic mutations have been identified in patients with NSCLC. The first genetic aberration was EGFR mutation, found in around 10-15\% of NSCLC cases in the US population [3]. However, in East Asia, an estimated $35 \%$ of people test positive for EGFR mutation. EGFR plays a role in the proliferation and survival of cancer cells [3]. Other major mutations discovered to be associated with EGFR mutation are the deletion of exon 19 or exon 21 (L858R) [6].

Tyrosine kinase inhibitors of the epidermal growth factor receptor (EGFR-TKIs) are a group of drugs that target the EGFR mutation and stimulate the immune system to kill cancer cells [7]. Erlotinib was the first EGFRTKI to be approved by the US FDA for use as a first-line treatment in advanced NSCLC with EGFR mutant harboring NSCLC [8]. Subsequently, other EGFR-TKIs, afatinib and gefitinib, were approved, in July 2013 and July 2015, respectively [8]. In some patients, however, cancer cells undergo secondary mutations which allow them to develop resistance to these drugs [7]. The most common secondary mutation is T790M [7]. Osimertinib is a

Future Medicine 
third generation EGFR-TKI that irreversibly binds to common mutations (19 deletion or exon 21) and to the secondary mutation that develops after resistance to the first and second generation EGFR-TKIs (i.e., the T790M mutation) [7]. Osimertinib was approved in March 2017 as a second-line treatment in the event of resistance to other EGFR-TKIs [9]. Then, based on the results of FLAURA trial, which compared osimertinib to erlotinib or gefitinib in the first-line treatment setting, osimertinib was approved by the FDA for use as a first-line treatment for patients with advanced EGFR + ve NSCLC [10].

There are five EGFR-TKIs (erlotinib, gefitinib, afatinib, osimertinib and dacomitinib) and all are first-line options for patients with EGFR +ve NSCLC [4,5,11]. Although most clinical trials used standard chemotherapy as a comparator [12,13], some trials have compared EGFR-TKIs to other EGFR-TKIs $[10,14,15]$. However, not all EGFRTKIs have been subject to such head-to-head comparisons. Thus, a network meta-analysis (NMA) is required to simultaneously compare EGFR-TKIs both, directly and indirectly, to guide clinicians in choosing between these treatment options. Therefore, we conducted a NMA to assess the comparative efficacy and safety of EGFR-TKIs as first-line treatments in patients with advanced or metastatic (Stage III and IV) EGFR + ve NSCLC, compared with standard chemotherapy or to each other.

\section{Materials \& methods}

The study was conducted in compliance with the Preferred Reporting Items for Systematic Reviews and MetaAnalyses extension statement for reporting of systematic reviews incorporating NMAs of healthcare interventions [16].

\section{Inclusion criteria}

Randomized clinical trials (RCTs) that compared erlotinib, afatinib, gefitinib, dacomitinib and osimertinib (as a single agent) to cytotoxic chemotherapy (including docetaxel and pemetrexed or mixtures of cytotoxic drugs) or to other EGFR-TKIs were included. Only RCTs in untreated patients were considered. Trials that included participants with unknown status of EGFR mutation (wild-type) were excluded. The data for the EGFR +ve population were extracted if it was reported separately in mixed population trials.

The primary outcomes assessed in the eligible trials are overall survival (OS), defined as the time from randomization to death from any cause [3]; progression-free survival (PFS), defined as the time from randomization to disease progression or death from any cause [17] and toxicity, measured by the number of participants with adverse events of grade 3 or higher, where adverse events were defined according to the Common Terminology Criteria for Adverse Events, available on the national cancer institute website [18]. The secondary outcome was the objective response rate (ORR), defined as the proportion of patients with a reduction in tumor burden of a predefined amount [19].

\section{Search methods \& study selection}

The Cochrane Central Register for Controlled Trials (CENTRAL), MEDLINE accessed via PubMed, Embase and clinicaltrials.gov were searched from their inception to December 2019. We restricted articles to those published in English language. The detailed search strategies for the databases searched are illustrated in (Supplementary Table 1). Each title and abstract were independently examined by two reviewers and irrelevant studies were excluded. Then, full-text screening was performed. Any disagreement between the two reviewers was resolved through discussion with a third reviewer.

\section{Data extraction \& management}

We extracted data using a standardized form in Microsoft Excel. The following data were extracted from each included trial: Trial characteristics: phase, single center versus multicenter, number of randomized patients and blind versus open-label. Details of treatment: drugs, dosage form, dose, and frequency. Patient characteristics: age, gender, stage, histology (nonsquamous vs squamous cell carcinoma), ethnicity (Asian vs Caucasian or others), performance status (PS) (PS 0-1 vs PS 2), history of smoking and EGFR mutation status (harboring vs wild-type or unknown EGFR status). Outcome data: hazard ratios (HRs) for PFS and OS and their 95\% CI, number of patients with an objective response, and number of patients with adverse events (AE) of grade 3 or higher.

\section{Assessment of risk of bias in included studies}

The risk of bias was assessed using the risk of bias tool described in the Cochrane Handbook for Systematic Reviews of Interventions [20]. The risk of bias was assessed by using the six evidence-based domains: selection 
bias, performance bias, detection bias (blinding of outcome assessment), attrition bias (incomplete outcome data), reporting bias (selective outcome reporting) and other forms of bias. For each risk of bias, 'low risk of bias', 'high risk of bias' or 'unclear risk of bias were assigned (Supplementary Table 2) [20].

\section{Data synthesis \& evaluation of statistical assumptions}

Pairwise meta-analysis

For each outcome, summary estimates of treatment effects for each comparison were calculated along with their 95\% CI. For time-to-event end points (OS, PFS), HRs were reported. When time-to-event data were unavailable from trial reports, individual survival patient data from published Kaplan-Meier curves was reconstructed and HRs were estimated [21]. For dichotomous outcomes (ORR and AEs), odds ratios (ORs) were estimated [21]. The total number of grade 3 and $4 \mathrm{AEs}$ in each treatment arm were calculated for each toxicity category [21]. The pair-wise meta-analysis was conducted using fixed-effect and random-effects models [3]. Statistical heterogeneity among trials were assessed by analyzing the forest plots, using the chi-square test $(\mathrm{p}<0.1$ was considered significant) and calculating the $\mathrm{I}^{2}$ statistics [21]. Stata Statistical Software was used for the meta-analysis (Version 15.1; StataCorp, TX, USA) [22].

\section{Network meta-analysis}

We conducted this NMA using a multivariate meta-analysis approach with the network package in Stata Statistical Software (Version 15.1; StataCorp) [22]. This method makes it possible to compare all drugs, even if there is no head-to-head clinical trial, as long as there is a common comparator. Before the pooling of studies in this NMA, we ensured that the transitivity assumption was met by qualitatively examining the distribution of patient and study characteristics that could modify treatment effects and ensure they are comparable across trials [23]. Consistency assumption was tested using both the loop-specific and side-splitting approaches [23-25]. Where both loop-specific and side-splitting tests suggested consistency between direct and indirect estimates for a particular outcome, we fit a consistency model (Supplementary Table 4).

Indirect estimates of the outcomes were calculated using the technique described by Bucher $e t$ al. [26]. Relative ORs for dichotomous outcomes and HRs for time-to-event outcomes were shown in the results for each possible comparison in the network. Furthermore, treatments were ranked using the surface under the cumulative ranking curve approach (SUCRA). SUCRA shows the percentage, or probability, of the effectiveness and safety of each treatment being ranked the best without uncertainty in comparison to an imaginary treatment that will always rank the first [17].

\section{Results}

Included studies

A total of 1389 articles (446 PubMed, 818 Embase and 125 Cochrane) were identified. After duplicates were removed, 650 articles remained.. After title and abstract screening, 105 articles were selected as relevant to our criteria. After full-text screening 15 RCTs were eligible for NMA with a total of 3750 participants (Figure 1) [10,12,14,15,27-35]. Of these studies, only 11 were included in the pairwise meta-analysis, while four were excluded because they contain only one study for a unique pair of TKIs (ARCHER1050, FLAURA, LUX-LUNG 7 and GTO0901) [10,15,36].

\section{Population characteristics}

Eleven of the 15 RCTs included participants with only EGFR +ve NSCLC. Four trials included a mixed population $(E G F R+\mathrm{ve},-\mathrm{ve}$ or wild-type), but in each case, the EGFR +ve population was reported in subgroups [28,29,31,32]. The majority of patients were of Asian ethnicity, although the EURTAC trial included only a Caucasian population [37]. All included trials reported median age for each arm except the trials that had mixed population. The median age ranged from 56 to 64 years. The percentage of females ranged from 53.1 to $70 \%$. The never smoker percentage was reported in 12 of the 15 studies, where the percentage ranged from 57.9 to $82 \%$ (Table 1). The main outcomes of the included studies are summarized in Supplementary Table 3.

\section{Risk of bias}

The results of risk of bias assessment for the eligible studies are summarized in Supplementary Figure 1. All the included studies had low risk of bias in terms of random sequence generation and allocation concealment, except for three and four studies, respectively. Thirteen of the 15 included studies had an open-label design, while the 


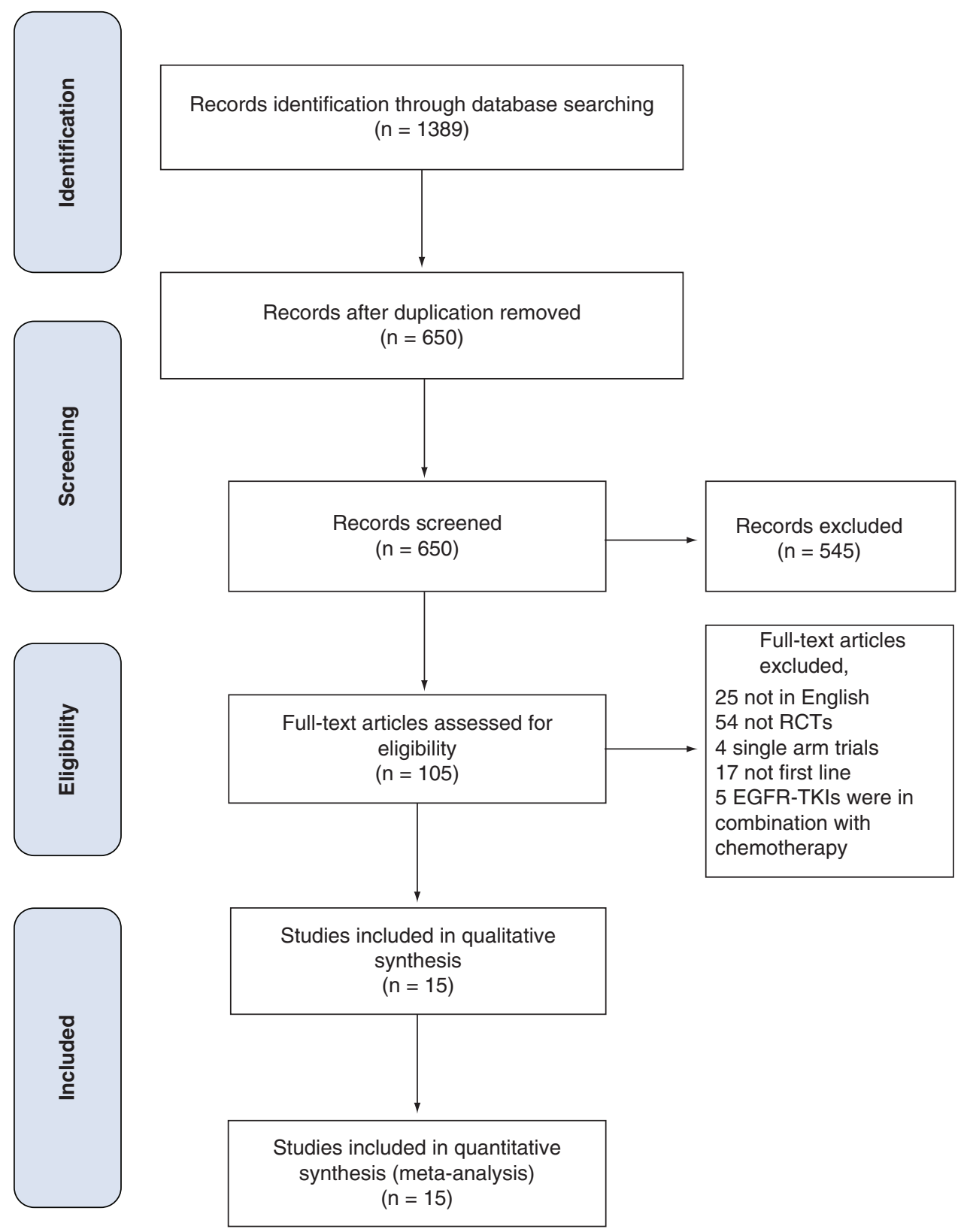

Figure 1. PRISMA flow diagram of search and study selection. Shows database searched, number of articles found, duplicates removed and subsequent processes leading to the selection of eligible studies.

EGFR-TKI: Tyrosine kinase inhibitors of the epidermal growth factor receptor; RCT: Randomized control trial.

majority had the primary outcome assessed by an independent reviewer. Overall, the eligible studies had low risks of attrition and reporting biases.

Pairwise meta-analysis findings

Erlotinib versus chemotherapy

Data from five trials were available for PFS [27,28,37-39]. The pooled effect estimate for PFS was $(\mathrm{HR}=0.44$, 95\% CI: 0.25-0.77; $\mathrm{I}^{2}=86.8 \%$ ), indicating a significant difference between the two groups in favor of erlotinib (Supplementary Figure 2). Data for OS were available from five trials. The pooled effect estimate (HR $=1.11,95 \%$ CI: $0.9-1.37 ; \mathrm{I}^{2}=0 \%$ ) indicated that no significant difference between the two arms (Supplementary Figure 3). 


\begin{tabular}{|c|c|c|c|c|c|c|c|c|}
\hline Study & Comparison & $\begin{array}{l}\text { Control } \\
\text { group }\end{array}$ & $\begin{array}{l}\text { Intervention } \\
\text { group }\end{array}$ & $\begin{array}{l}\text { Median age } \\
\text { control }\end{array}$ & $\begin{array}{l}\text { Median age } \\
\text { intervention }\end{array}$ & $\begin{array}{l}\text { Female } \\
(\%)\end{array}$ & $\begin{array}{l}\text { Never } \\
\text { smoker (\%, } \\
\mathrm{Cl})\end{array}$ & $\begin{array}{l}\text { Mutation distribution } \\
19 \mathrm{del} / \text { L858R/OTHER (\%) }\end{array}$ \\
\hline WJT0G3405 & Gefitinib vs chemotherapy & 86 & 86 & 64 & 64 & $69 \%$ & $71 \%$ vs 66 & $58 / 42 / 0$ vs $43 / 57 / 0$ \\
\hline NEJ002 & Gefitinib vs chemotherapy & 114 & 114 & 63.9 & 62.6 & $64 \%$ & $65.8 \%, 57.9$ & $\begin{array}{l}50.9 / 43 / 6.1 \text { vs } \\
51.8 / 42.1 / 6.1\end{array}$ \\
\hline EURTAC & Erlotinib vs chemotherapy & 86 & 87 & 63 & 64 & $72.50 \%$ & 66,72 & $66 / 34 / 0$ vs $67 / 33 / 0$ \\
\hline OPTIMAL & Erlotinib vs chemotherapy & 72 & 82 & 57 & 59 & $59.50 \%$ & 72,69 & $52 / 48 / 0$ vs $54 / 46 / 0$ \\
\hline IPASS & Gefitinib vs chemotherapy & 129 & 132 & NR & NR & NR & NR & NR \\
\hline ENSURE & Erlotinib vs chemotherapy & 110 & 107 & 57.5 & 56 & $61.25 \%$ & $71.8,69.2$ & $52.3 / 47.7 / 0$ vs $57 / 43 / 0$ \\
\hline Lux-lung3 & Afatinib vs chemotherapy & 115 & 230 & 61.5 & 61 & $65.50 \%$ & $67.4,70.4$ & $\begin{array}{l}49.1 / 39.6 / 11.3 \text { vs } \\
49.6 / 40.9 / 9.6\end{array}$ \\
\hline Lux-lung6 & Afatinib vs chemotherapy & 122 & 242 & 58 & 58 & $66 \%$ & $74.8,81.1$ & $\begin{array}{l}51.2 / 38 / 10.7 \text { vs } \\
50.8 / 37.7 / 11.5\end{array}$ \\
\hline Lux-lung7 & Afatinib vs gefitinib & 159 & 160 & 63 & 63 & $62 \%$ & 66,67 & $42 / 58 / 0$ vs $42 / 58 / 0$ \\
\hline ARCHER 1050 & Dacomitinib vs gefitinib & 225 & 227 & 62 & 61 & $60 \%$ & 65,64 & $59 / 41 / 0$ vs $59 / 41 / 0$ \\
\hline CTONG0901 & Erlotinib vs gefitinib & 128 & 128 & NR & NR & $53.50 \%$ & 82 vs 72.7 & $57.8 / 42.2$ vs $57.8 / 42.2$ \\
\hline FLAURA & $\begin{array}{l}\text { Osimertinib vs gefitinib or } \\
\text { erlotinib }\end{array}$ & 277 & 279 & 64 & 64 & $63 \%$ & 65,63 & $57 / 35 / 0$ vs $56 / 32 / 0$ \\
\hline First-SIGNAL & Gefitinib vs chemotherapy & 16 & 26 & NR & NR & $69 \%$ & NR & NR \\
\hline GTWOWG & Erlotinib vs chemotherapy & 86 & 86 & 64 & 64 & $64 \%$ & $70.9,66.3$ & $58 / 42 / 0$ vs $43 / 57 / 0$ \\
\hline TORCH & Erlotinib vs chemotherapy & 19 & 20 & 64 & 64 & $72.50 \%$ & $71 \%, 66$ & NR \\
\hline
\end{tabular}

NR: Not reported.

\section{Gefitinib versus chemotherapy}

PFS data were available from four trials. The pooled effect estimate (HR $=0.43,95 \%$ CI: $\left.0.33-0.55 ; \mathrm{I}^{2}=41 \%\right)$, indicated a significant difference between gefitinib and cytotoxic chemotherapy favoring gefitinib (Supplementary Figure 4). Data for OS were available from four trials (HR $=1,95 \% \mathrm{CI}: 0.83-1.2 ; \mathrm{I}^{2}=0 \%$ ) and showed no significant difference between the two arms (Supplementary Figure 5).

\section{Afatinib versus chemotherapy}

PFS data were available from two trials comparing afatinib with cytotoxic chemotherapy. The pooled effect estimate (HR $=0.4,95 \%$ CI: $\left.0.2-0.83 ; \mathrm{I}^{2}=90.2 \%\right)$ revealed a significant difference between afatinib and cytotoxic chemotherapy favoring afatinib (Supplementary Figure 6). OS data were available from the same two trials. The pooled effect estimate ( $\mathrm{HR}=0.91,95 \% \mathrm{CI}: 0.75-1.1 ; \mathrm{I}^{2}=0 \%$ ) showed no significant difference between the two groups (Supplementary Figure 7).

\section{Combined EGFR-TKIs versus chemotherapy}

Eleven trials compared EGFR-TKIs as a single agent with cytotoxic chemotherapy, comprising a total of 2235 participants. When we pooled all these trials together and considered all EGFR-TKIs as one arm and cytotoxic chemotherapy as the comparator, the pooled effect estimate for PFS and OS were: (HR $=0.43,95 \%$ CI: $0.33-$ $\left.0.55 ; \mathrm{I}^{2}=78.1 \%\right)$ and $\left(\mathrm{HR}=1,95 \% \mathrm{CI}: 0.89-1.12 ; \mathrm{I}^{2}=0 \%\right)$, respectively (Supplementary Figures $8 \& 9$ ). These results indicated significant difference in PFS favoring EGFR-TKIs, when compared with chemotherapy, while no significant difference was observed in OS between EGFR-TKIs and chemotherapy.

For ORR, all included studies with EGFR-TKIs were combined as one arm versus chemotherapy. The OR for each included trial was calculated and then pooled together to arrive at an estimation of the pooled ORR. The pooled OR of ORR was 4.49 (95\% CI: 3.16-6.37; $\mathrm{I}^{2}=64.9 \%$ ), which indicated that EGFR-TKIs are 4.49-times better than chemotherapy in respect to the tumor objective response (Supplementary Figure 10).

NMA findings

Overall survival

The HRs for OS were calculated between all treatments in the NMA. Table 2A shows the results for OS outcomes. All EGFR-TKIs performed better than chemotherapy, with statistically significant improvement in OS. The 
Table 2. League table summarizing treatment comparisons for efficacy and safety outcomes.

\begin{tabular}{|c|c|c|c|c|c|}
\hline \multicolumn{6}{|c|}{ A. League table comparing the overall survival hazard ratios of tyrosine kinase inhibitors for EGFR-mutant NSCLC ${ }^{\dagger}$} \\
\hline Treatment & Osimertinib & Gefitinib & Erlotinib & Dacomitinib & Chemotherapy \\
\hline Afatinib & $1.50(0.68,3.30)$ & $0.67(0.43,1.05)$ & $1.17(0.71,1.94)$ & $1.44(0.65,3.22)$ & $0.32(0.21,0.47)^{\ddagger}$ \\
\hline \multicolumn{2}{|l|}{ Osimertinib } & $0.45(0.23,0.86)^{\ddagger}$ & $0.78(0.36,1.69)$ & $0.96(0.38,2.45)$ & $0.21(0.10,0.44)^{\ddagger}$ \\
\hline \multicolumn{2}{|l|}{ Gefitinib } & & $1.74(1.15,2.62)^{\ddagger}$ & $2.14(1.09,4.18)^{\ddagger}$ & $0.47(0.34,0.65)^{\ddagger}$ \\
\hline \multicolumn{2}{|l|}{ Erlotinib } & & & $1.23(0.56,2.70)$ & $0.27(0.19,0.38)^{\ddagger}$ \\
\hline \multicolumn{2}{|l|}{ Dacomitinib } & & & & $0.22(0.11,0.46)^{\ddagger}$ \\
\hline \multicolumn{6}{|c|}{ B. League table comparing the progression-free survival hazard ratios of tyrosine kinase inhibitors for EGFR-mutant NSCLC ${ }^{\dagger}$} \\
\hline Treatment & Osimertinib & Gefitinib & Erlotinib & Dacomitinib & Chemotherapy \\
\hline Afatinib & $1.04(0.44,2.50)$ & $0.66(0.40,1.08)$ & $1.07(0.61,1.85)$ & $1.19(0.49,2.86)$ & $0.55(0.35,0.86)^{\ddagger}$ \\
\hline \multicolumn{2}{|l|}{ Osimertinib } & $0.63(0.31,1.30)$ & $1.02(0.44,2.38)$ & $1.14(0.41,3.17)$ & $0.53(0.24,1.18)$ \\
\hline \multicolumn{2}{|l|}{ Gefitinib } & & $1.62(1.04,2.52)^{\ddagger}$ & $1.80(0.87,3.72)$ & $0.83(0.58,1.19)$ \\
\hline \multicolumn{2}{|l|}{ Erlotinib } & & & $1.11(0.48,2.60)$ & $0.51(0.36,0.73)^{\ddagger}$ \\
\hline \multicolumn{2}{|l|}{ Dacomitinib } & & & & $0.46(0.21,1.04)$ \\
\hline \multicolumn{6}{|c|}{ C. League table comparing the objective response rate odds ratios of tyrosine kinase Inhibitors for EGFR-mutant NSCLC $\$$} \\
\hline Treatment & Osimertinib & Gefitinib & Erlotinib & Dacomitinib & Chemotherapy \\
\hline Afatinib & $1.29(0.48,3.50)$ & $1.61(0.91,2.84)$ & $1.18(0.60,2.30)$ & $1.39(0.51,3.78)$ & $5.69(3.40,9.54) \mathbb{I}$ \\
\hline \multicolumn{2}{|l|}{ Osimertinib } & $1.25(0.55,2.82)$ & $0.91(0.34,2.43)$ & $1.07(0.34,3.43)$ & $4.40(1.76,11.01)$ \\
\hline \multicolumn{2}{|l|}{ Gefitinib } & & $0.73(0.42,1.26)$ & $0.86(0.38,1.97)$ & $3.53(2.33,5.36)^{\mathbb{I}}$ \\
\hline \multicolumn{2}{|l|}{ Erlotinib } & & & $1.18(0.44,3.17)$ & $4.84(3.03,7.71)^{\mathbb{I}}$ \\
\hline \multicolumn{2}{|l|}{ Dacomitinib } & & & & $4.10(1.63,10.32)$ \\
\hline \multicolumn{6}{|c|}{ D. League table comparing the grade 3 or 4 adverse events odds ratios of tyrosine kinase inhibitors for EGFR-mutant NSCLC $\S$} \\
\hline Treatment & Osimertinib & Gefitinib & Erlotinib & Dacomitinib & Chemotherapy \\
\hline Afatinib & $5.99(0.79,45.39)$ & $4.29(1.40,13.10)$ & $3.22(0.93,11.13)$ & $1.48(0.19,11.73)$ & $0.75(0.27,2.10)$ \\
\hline \multicolumn{2}{|l|}{ Osimertinib } & $0.72(0.13,3.87)$ & $0.54(0.08,3.74)$ & $0.25(0.02,2.80)$ & $0.13(0.02,0.80) \mathbb{I}$ \\
\hline \multicolumn{2}{|l|}{ Gefitinib } & & $0.75(0.29,1.95)$ & $0.35(0.06,1.97)$ & $0.18(0.08,0.37)^{\mathbb{I}}$ \\
\hline \multicolumn{2}{|l|}{ Erlotinib } & & & $0.46(0.06,3.36)$ & $0.23(0.11,0.49)^{\mathbb{I}}$ \\
\hline \multicolumn{3}{|l|}{ Dacomitinib } & & & $0.51(0.08,3.37)$ \\
\hline \multicolumn{6}{|c|}{$\begin{array}{l}\text { †Comparison are based on hazard ratios estimates between tre } \\
\text { defining-treatment and row defining treatment. } \\
\text { ¥Significant results ( } 95 \% \mathrm{Cl} \text { for hazard ratio does not include 1). } \\
{ }^{\S} \text { Comparison are based on odds ratios estimates between treatm } \\
\text { treatment and row defining treatment. } \\
\text { I Significant results ( } 95 \% \mathrm{CI} \text { for odds ratio does not include 1). } \\
\text { EGFR: EGF receptor; NSCLC: Non-small-cell lung cancer. }\end{array}$} \\
\hline
\end{tabular}

comparison between gefitinib and other EGFR-TKIs showed erlotinib, dacomitinib and osimertinib significantly improved OS compared to gefitinib; whereas no difference was observed with afatinib. No statistically significant difference was observed when comparing afatinib, erlotinib, dacomitinib, and osimertinib to each other.

\section{Progression-free survival}

We present HRs for PFS among all the drug comparisons in this NMA (Table 2B). The network graph for the estimated comparisons is illustrated in Figure 2. In cases where no direct comparison between treatments exists, HRs were estimated indirectly. In cases where there have been direct head-to-head trials, the HRs reported here were a composite of direct and indirect comparisons. In comparison to chemotherapy, erlotinib $(\mathrm{HR}=0.51$, 95\% CI: $0.36,0.73)$ and afatinib (HR $=0.55,95 \%$ CI: $0.35,0.86)$ improved PFS respectively. In the case of gefitinib, dacomitinib, and osimertinib improved PFS; however, the improvements were not statistically significant. Comparing gefitinib to other EGFR-TKIs, erlotinib significantly improved PFS only against gefitinib ( $\mathrm{HR}=0.62$, $95 \%$ CI: 0.40, 0.96). No significant differences were observed with the remaining comparisons of EGFR-TKIs.

\section{Adverse events}

Table 2D presents the ORs for each possible comparison in the NMA. For each included clinical trial, adverse events at grade 3 or higher were summarized in (Supplementary Table 3). In comparison to chemotherapy, erlotinib (ORs 
Figure 2. Network plot of included trials. Network plot showing the drugs that were compared in the network meta-analysis. Drugs that connected with lines were compared directly and indirectly in the analysis. If there is no line between two drugs, it means no direct comparison exist in trials and HR's were estimated indirectly in the network meta-analysis.

HR: Hazard ratio.

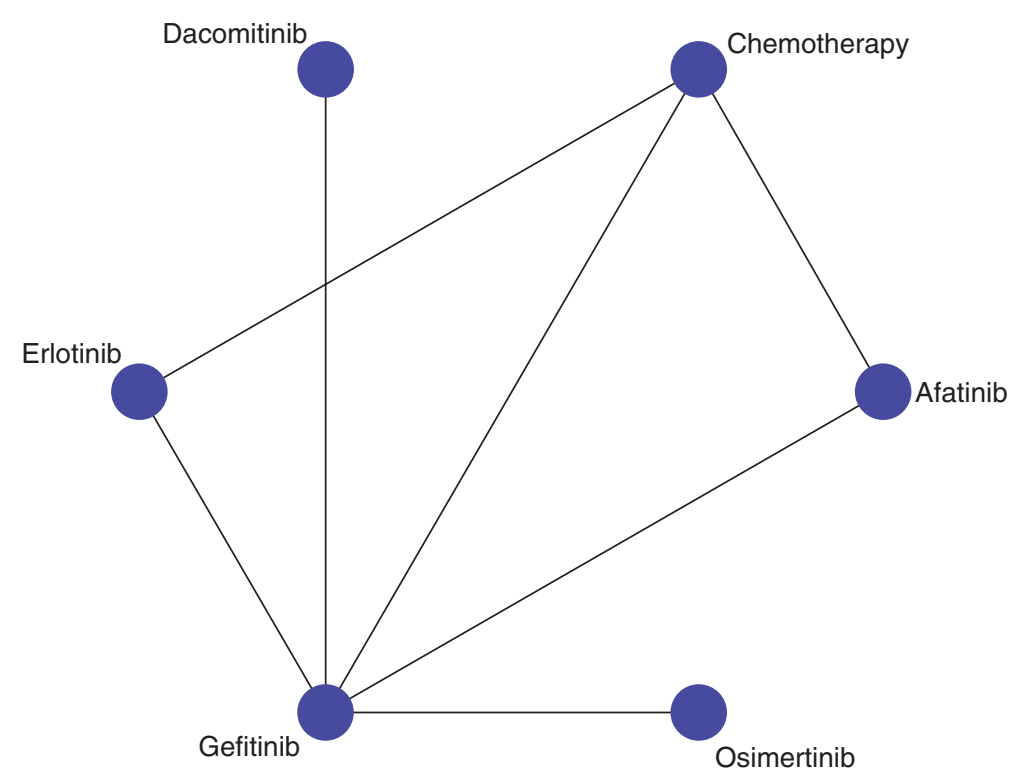

$=0.23,95 \%$ CI: 0.11, 0.49), gefitinib $(\mathrm{OR}=0.1895 \%$ CI: 0.08, 0.37), and osimertinib 0.13, 95\% CI: 0.02, 0.80 had significantly lower odds of grade 3 or higher AEs. Comparing the differences in grade 3 or higher AEs between the various EGFR-TKIs, we observed differences between the odds of AEs associated with various EGFR-TKIs, but the only significant difference was between gefitinib and afatinib. Specifically, afatinib had significantly greater odds of grade 3 or higher AEs compared with gefitinib (OR = 4.29, 95\% CI: 1.40, 13.10).

\section{Secondary outcomes}

Objective response rate

Table 2C shows the NMA results for the ORR outcome. In comparison to chemotherapy, all EGFR-TKIs showed significant improvement in ORR: afatinib $(\mathrm{OR}=5.69,95 \% \mathrm{CI}: 3.40,9.54)$, dacomitinib $(\mathrm{OR}=4.10,95 \%$ CI: $1.63,10.32)$, erlotinib $(\mathrm{OR}=4.84,95 \% \mathrm{CI}: 3.03,7.71)$, gefitinib $(\mathrm{OR}=3.53,95 \% \mathrm{CI}: 2.33,5.36)$, and osimertinib (OR $=4.40,95 \% \mathrm{CI}: 1.76,11.01)$. No statistically significant difference in ORR was observed when the EGFR-TKIs were compared among each other.

\section{Ranking}

PFS results suggests that dacomitinib had highest probability of being the most effective (SUCRA, 75.9\%), followed by, osimertinib (SUCRA, 69.5\%), erlotinib (SUCRA, 59.9\%), afatinib (SUCRA, 55.9\%), gefitinib (SUCRA, 36.1\%), chemotherapy (SUCRA, 2.7\%). We observed a similar ranking trend for OS except for the fact that osimertinib was ranked first while dacomitinib ranked second. AE results suggest that osimertinib (SUCRA, 84.3\%) and gefitinib (SUCRA, 78.9\%) has the highest probability of being the safest (Figure 3). Cluster plots were created for OS and safety, as well as ORR and safety, as shown in Figure 4. According to the cluster plot, osimertinib was the best treatment option when OS and safety are considered. As expected, chemotherapy occupied the left lower quadrant. This implies that chemotherapy is the least efficacious treatment and it subjects the patients to the highest incidence of adverse events. Afatinib was ranked first in terms of ORR, but in safety it was ranked fourth. Osimertinib was ranked second in ORR and first in safety. Overall, osimertinib was ranked highest.

\section{Discussion}

For patients with EGFR + ve NSCLC, first and second-generation EGFR-TKIs (erlotinib, gefitinib and afatinib) significantly improved PFS when compared with chemotherapy according to RCTs $[12,27,30]$. Afatinib, dacomitinib and osimertinib were compared with gefitinib or erlotinib in first-line treatment setting and all showed significant improvement in PFS $[10,15,36]$. This NMA showed significant improvement in PFS for both afatinib and erlotinib with chemotherapy. When comparing erlotinib with gefitinib, erlotinib significantly improved PFS than gefitinib. All EGFR-TKIs significantly improved OS in comparison with chemotherapy. Additionally, erlotinib, dacomitinib and osimertinib significantly improved OS when compared with gefitinib. ORR results showed that all EGFR-TKIs 

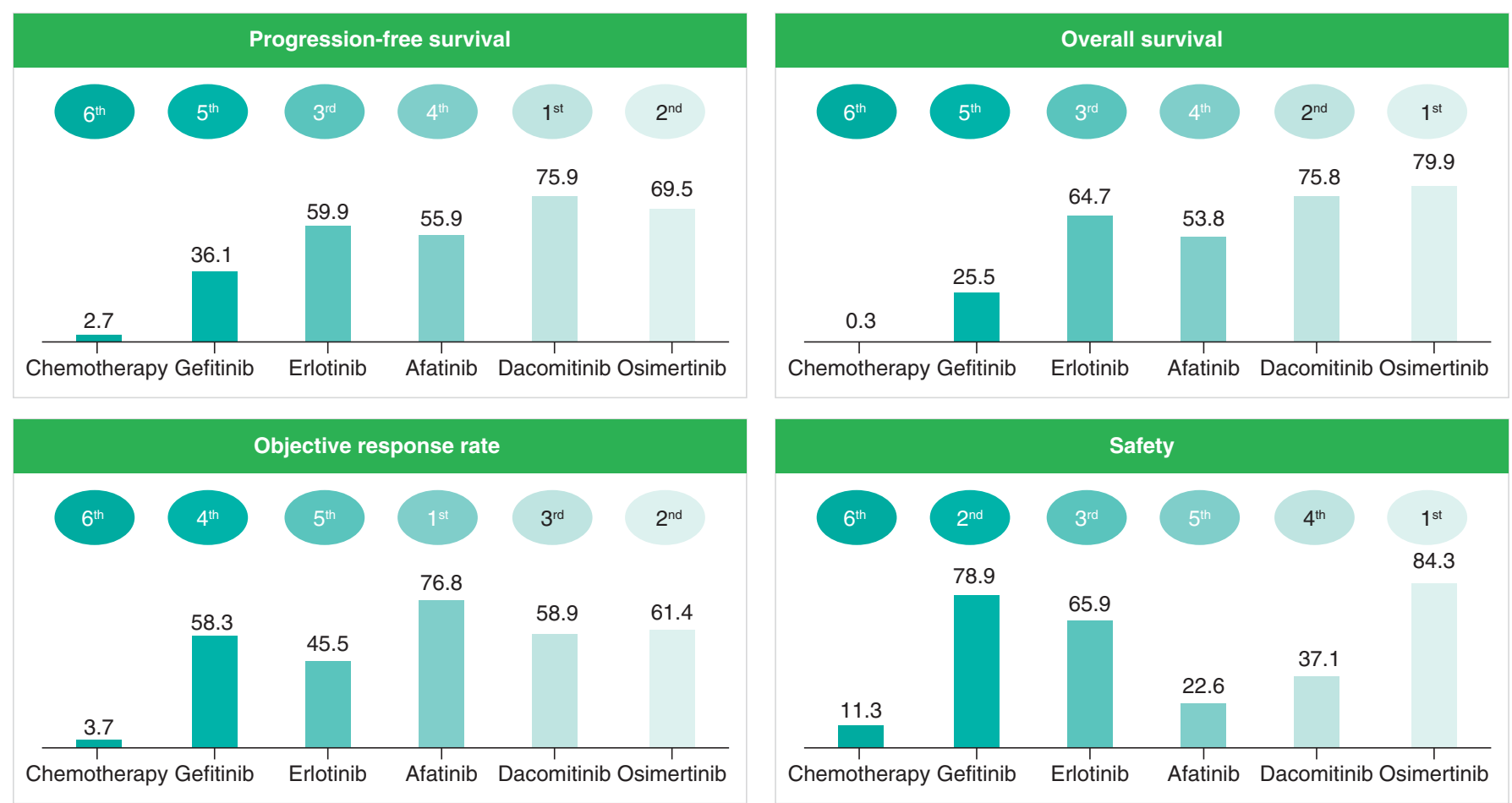

Figure 3. Surface under the cumulative ranking curve values for progression-free survival, overall survival, objective response rate and safety. SUCRA values showing the percentage of efficacy or safety for each treatment to be ranked the first compared with an imaginary treatment that will be ranked first without uncertainty. For each outcome, treatment with the highest SUCRA value is ranked the most efficacious or safest while treatment with the lowest SUCRA value is ranked the least efficacious or least safe.

SUCRA: Surface under the cumulative ranking curve.
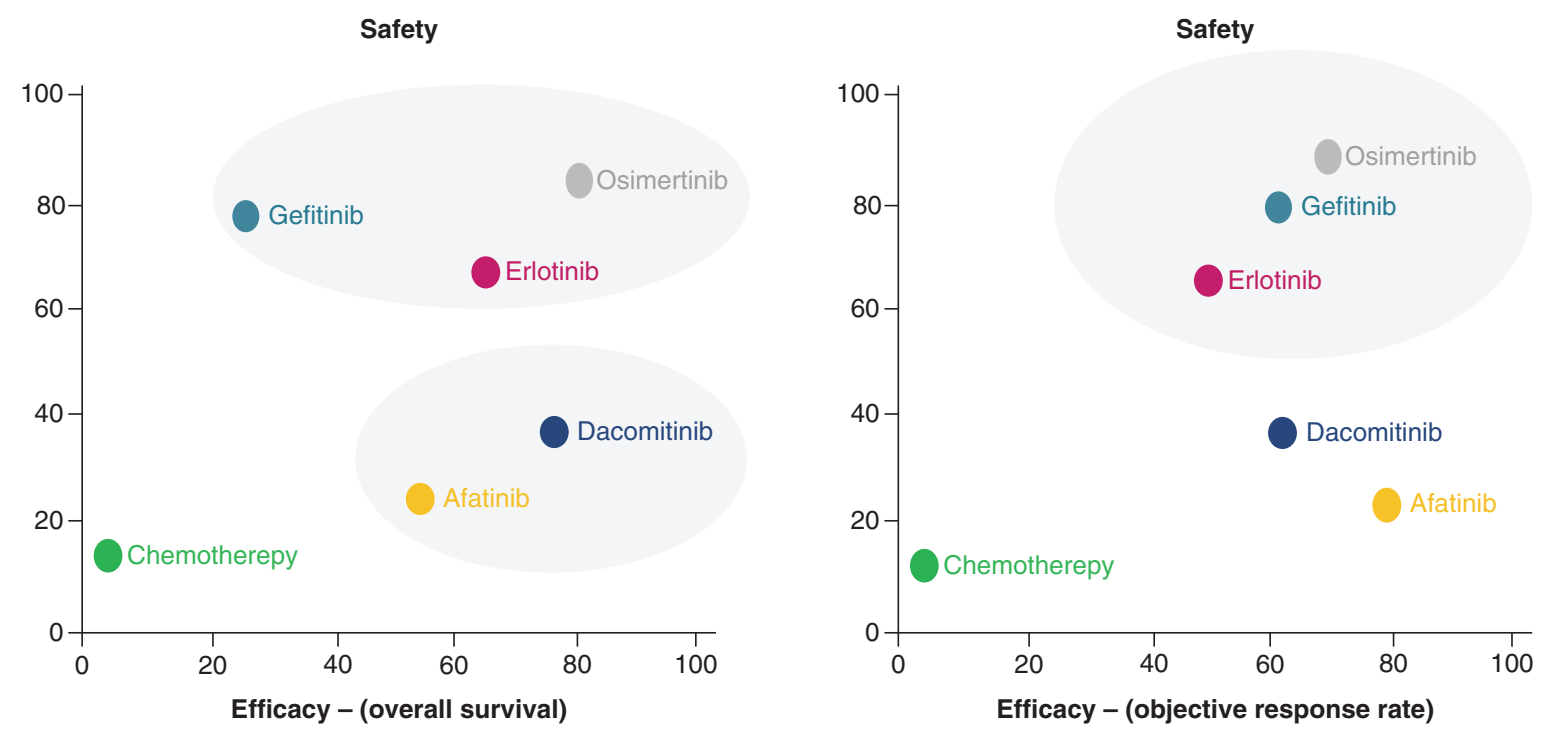

Figure 4. Cluster plot for overall survival (OS) and cluster plot for objective response rate. Plot shows SUCRA values on a scale of $0-100 \%$ for two outcomes. Each oval shap represents a group of treatments that belongs to the same cluster. The upper right quadrant represents the more effective and more safe treatments; lower right quadrant, more effective but less safe; lower left quadrant, less effective and less safe and upper left quadrant, less effective and safer. SUCRA: Surface under the cumulative ranking curve. 
were significantly better than chemotherapy in tumor shrinkage or objective response. When we compared the ORs of grade 3 or higher AEs for EGFR-TKIs, the result showed that erlotinib, gefitinib and osimertinib had a significantly better safety profile than chemotherapy. Gefitinib also showed significantly lower odds of grade 3 or higher AEs when compared with afatinib.

The NMA allowed us to compare all possible pairs even when there were no head-to-head trials. Efficacy and safety for all alternatives were estimated by direct and indirect comparison, thus giving the results more power. The total number of participants in the NMA was 3750, which is relatively high, given the low recruitment rate in cancer trials. In order to pick all eligible participants in this population, four trials that originally enrolled a mixed population $(E G F R+\mathrm{VE}$, -ve or wild-type) were included and the data for EGFR + ve population from the subgroup analyses were extracted $[28,29,31]$. This allowed use to include more studies than other NMAs of TKIs in EGFR mutant NSCLC [41].

Pairwise meta-analysis was unable to identify any significant improvement in OS when comparing EGFR-TKIs as a group with chemotherapy. Although most individual trials showed that EGFR-TKIs performed better than chemotherapy in OS, these individual results did not reach significance. The ability to combine direct and indirect comparisons in a NMA enabled us to identify that in fact erlotinib, afatinib and osimertinib all exhibited significant improvement in OS versus chemotherapy. When gefitinib was compared with chemotherapy, however, there was no significant improvement in terms of OS and this finding is reasonable. Three included RCTs compared erlotinib, afatinib and osimertinib with gefitinib and while all these trials showed an improvement in OS with the latter drug, this improvement was not significant. The NMA suggested that gefitinib exhibited the lowest improvement in OS of all the EGFR-TKIs.

In comparison to other published NMAs, this NMA included more trials and thus had a bigger sample size. Additionally, a NMA published in 2018 only reported PFS as efficacy outcome and did not report any ORR or OS results, while all outcomes were included in this NMA [40]. Erlotinib and gefitinib were combined in one arm in the previously published NMA, while they were analyzed separately in this NMA [40]. Further, only the PFS ranking scores were reported in the previously published article [40]. Notably, we reported ranking order for OS as well. The ranking order for PFS in our study showed that dacomitinib ranked first while the other NMA showed that osimertinib ranked first. The PFS results for our study were approximately consistent with the previous study in general, but our study did not show a significant difference in PFS when comparing osimertinib or dacomitinib with chemotherapy while the other study did. This difference can be explained on account of the fact that in the previous study erlotinib and gefitinib were analyzed as a single arm [40].

Another NMA for the use of EGFR-TKIs in the NSCLC and EGFR + ve population was published in 2014 [41]. This NMA included 11 RCTs encompassing erlotinib, gefitinib, afatinib and icotinib [41]. Icotinib is a EGFR-TKI that has been approved in China but not in the US [41]. That study concluded that EGFR-TKIs were significantly better than chemotherapy in terms of ORR and PFS, but they exhibited no significant difference in OS [41]. These results are consistent with ours except for OS. Our study showed that EGFR-TKIs led to a significant improvement in OS in comparison with chemotherapy. This difference is probably because our study included 15 trials with higher sample size and two more drugs were included in the NMA (osimertinib and dacomitinib). When the ranking order was compared with the previously published article, erlotinib was ranked first in ORR and PFS, while afatinib was ranked first in respect to 2-year OS in the previous study. In our study, the efficacy ranking showed that dacomitinib and osimertinib was ranked first in respect to PFS and OS, respectively, while afatinib was ranked first for ORR. Because osimertinib and dacomitinib were included in this NMA, the difference in ranking between our study and other previously published articles is reasonable.

SUCRA value allowed us to estimate the probability that a given EGFR-TKI would be the best for efficacy and safety, taking into account the magnitude of the effect and the uncertainty around it. According to SUCRA values, we created cluster plots that allowed us to visualize SUCRA ranking for efficacy (OS, PFS) and safety (grade 3 or higher AE). By simultaneously looking at efficacy and safety together, osimertinib has the most favorable benefit-risk profile among all EGFR-TKIs. Although dacomitinib was ranked first in terms of PFS, it was ranked fourth in terms of safety. A limitation of SUCRAs is that they pose challenges for interpretation and are not intuitive measures of effect. Despite their limitations, researchers have proposed alternative presentation formats that will likely be helpful for clinicians. As a result, we present SUCRA rankings in cluster plots to allow for simultaneous assessment of the benefits and risks of EGFR-TKIs. The cluster will enable clinicians to make decisions by comparing these treatments' efficacy and safety in a two-dimensional way. 
There are a few limitations to our study. First, the FLAURA trial compared osimertinib to either erlotinib or gefitinib, but the authors considered gefitinib and erlotinib as a single arm and did not report the results for each one alone [10]. As a result, gefitinib was considered as the control arm to make the analysis doable. Because osimertinib and dacomitinib were approved recently and there is only one RCT for each of them as a first-line option, the picture of the safety profile is not yet complete because some safety issues can appear months or years after approval.

\section{Conclusion}

The NMA concluded that all the included EGFR-TKIs were better than chemotherapy in terms of improving effectiveness and safety outcomes. Osimertinib was relatively the best option among this group according to the ranking order for efficacy and safety outcomes. These results may guide clinicians in choosing the most appropriate treatment option among EGFR-TKIs for their patient's individual clinical characteristics.

\section{Future perspective}

Targeted therapy and immunotherapy are fast developed discipline in oncology. In this specific patient's population, trials that evaluate the efficacy and safety of a combination of EGFR-TKIs and immunotherapy are expected in the future.

\section{Summary points}

- Tyrosine kinase inhibitors of the epidermal growth factor receptor (EGFR-TKIs), which include afatinib, dacomitinib, erlotinib, gefitinib, and osimertinib, are the standards of care for first-line treatment in patients with EGFR mutation-positive advanced NSCLC.

- Until the emergence of evidence on the efficacy and safety of dacomitinib and osimertinib, the first-generation EGFR-TKIs erlotinib and gefitinib were the standards of care.

- In clinical trials, dacomitinib and osimertinib significantly improved survival outcomes when compared to either erlotinib or gefitinib.

- In this network meta-analysis, all EGFR-TKIs improved both progression-free survival and overall survival with fewer grade 3 or higher adverse events in comparison to chemotherapy.

- Erlotinib, dacomitinib, and osimertinib significantly improved survival compared to gefitinib.

- This network meta-analysis suggests that osimertinib has the highest probability of being the most efficacious and the safest treatment option.

Supplementary data

To view the supplementary data that accompany this paper please visit the journal website at: www.futuremedicine.com/doi/suppl/10.2217/Imt-2020-0011

Acknowledgments

The authors would like to thank T Eguale for methodological advice.

Financial \& competing interests disclosure

The authors have no relevant affiliations or financial involvement with any organization or entity with a financial interest in or financial conflict with the subject matter or materials discussed in the manuscript. This includes employment, consultancies, honoraria, stock ownership or options, expert testimony, grants or patents received or pending, or royalties.

No writing assistance was utilized in the production of this manuscript.

\section{Open access}

This work is licensed under the Attribution-NonCommercial-NoDerivatives 4.0 Unported License. To view a copy of this license, visit http://creativecommons.org/licenses/by-nc-nd/4.0/

\section{References}

Papers of special note have been highlighted as: $\bullet$ of interest

1. Lung cancer in the world: the incidence, mortality rate and risk factors - WCRJ. https://www.wcrj.net/article/911

2. Siegel RL, Miller KD, Jemal A. Cancer statistics, 2015. CA. Cancer J. Clin. 65(1), 5-29 (2015). 
3. Greenhalgh J, Dwan K, Boland A et al. First-line treatment of advanced epidermal growth factor receptor (EGFR) mutation positive non-squamous non-small-cell lung cancer. Cochrane Database Syst. Rev. 5, CD010383 (2016).

4. Reck M, Popat S, Reinmuth N et al. Metastatic non-small-cell lung cancer (NSCLC): ESMO Clinical Practice Guidelines for diagnosis, treatment and follow-up. Ann. Oncol. 25(Suppl. 3), iii27-iii39 (2014).

5. Hanna N, Johnson D, Temin S et al. Systemic Therapy for Stage IV Non-Small-Cell Lung Cancer: American Society of Clinical Oncology Clinical Practice Guideline Update. J. Clin. Oncol. 35(30), 3484-3515 (2017).

6. Erlotinib Mechanism of Action - Clinical Pharmacology [Internet]. Elsevier, FL, USA. https://www-clinicalkey-com.ezproxymcp.flo.org/pharmacology/monograph $/ 2754$ ? sec=monmech

7. Sundaresan TK, Sequist LV, Heymach JV et al. Detection of T790M, the acquired resistance EGFR mutation, by tumor biopsy versus noninvasive blood-based analyses. Clin. Cancer Res. 22(5), 1103-1110 (2016).

8. Drugs@FDA: FDA Approved Drug Products. https://www.accessdata.fda.gov/scripts/cder/daf/

9. Yang JC-H, Ahn M-J, Kim D-W et al. Osimertinib in pretreated T790M-positive advanced non-small-cell lung cancer: AURA Study Phase II extension component. J. Clin. Oncol. 35(12), 1288-1296 (2017).

10. Soria J-C, Ohe Y, Vansteenkiste J et al. Osimertinib in untreated EGFR-mutated advanced non-small-cell lung cancer. N. Engl. J. Med. 378(2), 113-125 (2018).

11. Wood DE, Chair V, Aisner DL et al. NCCN Guidelines Version 3.2019 non-small-cell lung cancer (2019).

12. Sequist LV, Yang JC-H, Yamamoto $\mathrm{N}$ et al. Phase III study of afatinib or cisplatin plus pemetrexed in patients with metastatic lung adenocarcinoma with EGFR mutations. J. Clin. Oncol. 31(27), 3327-3334 (2013).

13. Katakami N, Atagi S, Goto K et al. LUX-Lung 4: a Phase II trial of afatinib in patients with advanced non-small-cell lung cancer who progressed during prior treatment with erlotinib, gefitinib, or both. J. Clin. Oncol. 31(27), 3335-3341 (2013).

14. Park K, Tan E-H, O'Byrne K et al. Afatinib versus gefitinib as first-line treatment of patients with EGFR mutation-positive non-small-cell lung cancer (LUX-Lung 7): a Phase IIB, open-label, randomised controlled trial. Lancet Oncol. 17(5), 577-589 (2016).

15. Wu Y-L, Cheng Y, Zhou X et al. Dacomitinib versus gefitinib as first-line treatment for patients with EGFR-mutation-positive non-small-cell lung cancer (ARCHER 1050): a randomised, open-label, Phase III trial. Lancet Oncol. 18(11), 1454-1466 (2017).

16. Hutton B, Salanti G, Caldwell DM et al. The PRISMA extension statement for reporting of systematic reviews incorporating network meta-analyses of health care interventions: checklist and explanations. Ann. Intern. Med. 162(11), 777 (2015).

17. Shim S, Yoon B-H, Shin I-S, Bae J-M. Network meta-analysis: application and practice using Stata. Epidemiol. Health 39, e2017047 (2017).

18. National Cancer Institute. Common Terminology Criteria for Adverse Events (CTCAE) | Protocol Development | CTEP [Internet]. https://ctep.cancer.gov/protocoldevelopment/electronic_applications/ctc.htm\#ctc_50

19. FDA. Clinical Trial End points for the Approval of Cancer Drugs and Biologics - Guidance for Industry (2018). http://www.liebertonline.com/doi/abs/10.1089/blr.2007.9941

20. Cochrane Cochrane Handbook for Systematic Reviews of Interventions | Cochrane Training [Internet].Cochrane. Higgins JPT, Thomas J, Chandler J et al. (Eds). https://training.cochrane.org/handbook

21. Créquit P, Yavchitz A, Trinquart L, Ravaud P, Cadranel J. Epidermal growth factor receptor (EGFR) Tyrosine Kinase Inhibitors versus single agent chemotherapy as second-line treatment for non-small-cell lung cancer wild-type or unknown status for EGFR. Cochrane Database Syst. Rev. (2018). http://doi.wiley.com/10.1002/14651858.CD012901

22. Sterne JAC, Bradburn MJ, Egger M. Meta-Analysis in Stata ${ }^{\top M}$ [Internet]. In: Systematic Reviews in Health Care BMJ Publishing Group, London, UK, 347-369 (2019).

23. Riley RD, Jackson D, Salanti G et al. Multivariate and network meta-analysis of multiple outcomes and multiple treatments: rationale, concepts, and examples. BMJ358, j3932 (2017).

24. White IR, Barrett JK, Jackson D, Higgins JPT. Consistency and inconsistency in network meta-analysis: model estimation using multivariate meta-regression. Res. Synth. Methods 3(2), 111-125 (2012).

25. Mavridis D, Salanti G. A practical introduction to multivariate meta-analysis. Stat. Methods Med. Res. 22(2), 133-158 (2013).

26. Bucher HC, Guyatt GH, Griffith LE, Walter SD. The results of direct and indirect treatment comparisons in meta-analysis of randomized controlled trials. J. Clin. Epidemiol. 50(6), 683-691 (1997).

27. Zhou C, Wu Y-L, Chen G et al. Erlotinib versus chemotherapy as first-line treatment for patients with advanced EGFR mutation-positive non-small-cell lung cancer (OPTIMAL, CTONG-0802): a multicentre, open-label, randomised, Phase III study. Lancet. Oncol. 12(8), 735-742 (2011).

28. Carter LR, Califano R. The TORCH trial. Transl. Lung Cancer Res. 1(4), 283-286 (2012).

29. Han J-Y, Park K, Kim S-W et al. First-SIGNAL: first-line single-agent iressa versus gemcitabine and cisplatin trial in never-smokers with adenocarcinoma of the lung. J. Clin. Oncol. 30(10), 1122-1128 (2012). 
30. Watanabe S, Inoue A, Nukiwa T, Kobayashi K. Comparison of gefitinib versus chemotherapy in patients with non-small-cell lung cancer with exon 19 deletion. Anticancer Res. 35(12), 6957-6961 (2015).

31. Fukuoka M, Wu Y-L, Thongprasert $S$ et al. Biomarker analyses and final overall survival results from a Phase III, randomized, open-label, first-line study of gefitinib versus carboplatin/paclitaxel in clinically selected patients with advanced non-small-cell lung cancer in Asia (IPASS). J. Clin. Oncol. 29(21), 2866-2874 (2011).

32. Mok TS, Wu Y-L, Thongprasert $S$ et al. Gefitinib or carboplatin-paclitaxel in pulmonary adenocarcinoma. N. Engl. J. Med. 361(10), 947-957 (2009).

33. Douillard J-Y, Ostoros G, Cobo M et al. First-line gefitinib in caucasian EGFR mutation-positive NSCLC patients: a Phase-IV, open-label, single-arm study. Br. J. Cancer 110(1), 55-62 (2014).

34. Ramalingam SS, Vansteenkiste J, Planchard D et al. Overall survival with osimertinib in untreated, EGFR-mutated advanced NSCLC. N. Engl. J. Med. 382(1), 41-50 (2020).

- Shows the improvement in overall survival when compare osimertinib with gefitinib or erlotinib this trial (FLAURA) changed the practice.

35. Mok TSK, Cheng Y, Zhou X et al. Updated overall survival (OS) from extended follow up in ARCHER 1050: a randomized Phase III study comparing dacomitinib with gefitinib as first-line therapy for patients (pts) with EGFR mutations. Ann. Oncol. 30, ix200-ix201 (2019).

- Shows the advantage of dacomitinib over gefitinib.

36. Park K, Tan E-H, O'Byrne K et al. Afatinib versus gefitinib as first-line treatment of patients with EGFR mutation-positive non-small-cell lung cancer (LUX-Lung 7): a Phase IIB, open-label, randomised controlled trial. Lancet Oncol. 17(5), 577-589 (2016).

37. Rosell R, Carcereny E, Gervais R et al. Erlotinib versus standard chemotherapy as first-line treatment for European patients with advanced EGFR mutation-positive non-small-cell lung cancer (EURTAC): a multicentre, open-label, randomised Phase III trial. Lancet Oncol. 13(3), 239-246 (2012).

38. Wu Y-L, Zhou C, Liam C-K et al. First-line erlotinib versus gemcitabine/cisplatin in patients with advanced EGFR mutation-positive non-small-cell lung cancer: analyses from the Phase III, randomized, open-label, ENSURE study. Ann. Oncol. 26(9), 1883-1889 (2015).

39. Reck M, Von Pawel J, Fischer JR et al. Erlotinib versus carboplatin/vinorelbine in elderly patients (age 70 or older) with advanced non-small-cell lung carcinoma (NSCLC): a randomized Phase II study of the German Thoracic Oncology Working Group. 28(Suppl. 15), 7565-7565 (2016).

40. Ma S-K, Li X-Y, Wu S-X, Lin J-Z, Yu S-H. A network meta-analysis of nonsmall-cell lung cancer patients with an activating EGFR mutation. Medicine (Baltimore) 97(30), e11569 (2018).

41. Liang W, Wu X, Fang W et al. Network meta-analysis of erlotinib, gefitinib, afatinib and icotinib in patients with advanced non-small-cell lung cancer harboring EGFR mutations. PLoS ONE 9(2), e85245 (2014). 